DESY $87-161$

December 1987

CALCULATION OF BEAM ENVELOPES IN STORAGE RINGS AND TRANSPORT SYSTEMS

IN THE PRESENCE OF TRANSVERSE SPACE CHARGE EFFECTS AND COUPLING

by

I. Borchardt, E. Karantzoulis, H. Mais, G. Ripken

Deutsches Elektronen-Sunchrotron DESY, Hamburg

ISSN 0418-9833 
DESY behält sich alle Rechte für den Fall der Schutzrechtserteilung und für die wirtschaftliche Verwertung der in diesem Bericht enthaltenen Informationen vor.

DESY reserves all rights for commercial use of information included in this report, especially in case of filing application for or grant of patents.

"Die Verantwortung für den Inhalt dieses

Internen Berichtes liegt ausschließlich beim Verfasser" 


\title{
Calculation of Beam Envelopes in Storage Rings and Transport Systems in the Presence of Transverse Space Charge Effects and Coupling
}

\author{
I. Borchardt, E. Karantzoulis, H. Mais, G. Ripken
}

August, 1987

\begin{abstract}
In the following report we describe a method for calculating the envelopes of coasting beams in linear coupled storage rings and transport systems in the presence of transverse space charge forces. This work is an extension of earlier calculations[2,3] to include coupled beam optics and energy deviations. The extension is achieved by defining a 5 dimensional ellipsoid in the $x-p_{x}-z-p_{z}-\Delta p / p$ space. The motion of this ellipsoid under the influence of the external fields and the instantaneous space charge forces can be described by five generating orbit vectors which can be combined into a 5 -dimensional matrix $\underline{B}(s)$. This "bunch -shape matrix", $\underline{B}(s)$, contains complete information about the configuration of the bunch. The solution of the equations of motion is carried through in the thin lens approximation.
\end{abstract}

\section{Contents}

1 Introduction $\quad 2$

2 Equations of Motion $\quad 2$

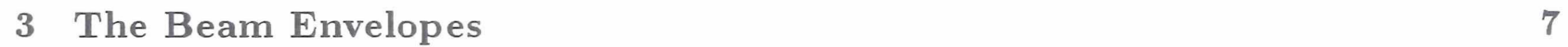

3.1 The 5 -dimensional ellipsoid in $x-p_{x}-z-p_{z}-\Delta p / p$ space . . . . . . . . 7

3.2 The projection of the five dimensional ellipsoid . . . . . . . . . . . . 9

3.2.1 Projection on the $x-z$ plane. . . . . . . . . . . . 10

3.2.2 Projection on the $x-p_{x}$ plane. . . . . . . . . . . . 14

3.2.3 Projection on the $z-p_{z}$ plane................ 15

4 Solution of the Equations of Motion $\quad 16$

4.1 Thin lens approximation . . . . . . . . . . . . . . . . 16

4.2 Initial conditions for the ellipsoid . . . . . . . . . . . . . . 19

5 Summary 21

6 Acknowledgements 22 


\section{Introduction}

In simple treatments of beam transport in storage rings and beam lines, one neglects electromagnetic interactions between the particles. The evolution of the beam envelope can then be calculated from a knowledge of the single particle motion as determined by the external guide field of the lens system.

This approximation is justified when the particles are highly relativistic. In this case the electrostatic and magnetic forces between pairs of particles essentially cancel since they have similiar magnitudes and opposite signs[1].

The position is different, however, when the particles are travelling slowly compared to the speed of light as, for example, in the case of heavy ions. Then, the electrostatic repulsion is stronger than the magnetic attraction and there is a net force on the particles over and above that due to the lens system.

When such a space charge force is present it is no longer possible to calculate beam envelopes in $x-p_{x}-z-p_{z}$ space using the independent particle approach; the motion of a single particle is influenced by the electromagnetic forces of all the remaining particles of the beam. Since these forces are on balance repulsive, the beam has a tendency to expand and particles can be lost if the physical machine aperture is not correspondingly increased.

In the following, we show how to calculate the envelopes and sizes of coasting beams in the presence of transverse space charge forces so that more accurate estimates of the required machine aperture can be made.

If the coupling between the betatron oscillations and the energy deviations of the particles is neglected the formalism developed here contains the results of J.M.Kapchinskij and V.V.Vladimirskij $[2,3]$ as a special case.

\section{Equations of Motion}

The calculation begins with the equation of motion of a particle of charge e:

$$
m_{0} \cdot \frac{d^{2}}{d t^{2}}(\gamma \cdot \vec{r})=\frac{e}{c}(\dot{\vec{r}} \wedge \vec{B})+\vec{F}^{\text {self }}
$$

where $\vec{B}$ is the external guide field and $\vec{F}^{\text {self }}$ is the space charge force due to the beam itself.

For simplicity, we make the following assumptions about the charge distribution of the bunch:

1.) We consider a coasting beam whence the longitudinal dimension is much greater than the transverse dimension. Only transverse forces are included.

2.)At position $s$, the beam has an ellipse-like cross section in the $x-z$ plane given by the equation $[7,8,9]$

$$
E_{z}^{2} \cdot x^{2}-2 E_{x} G_{x} \cdot x z+E_{x}^{2} \cdot z^{2}=\varepsilon_{x z}^{2},
$$

where $E_{x}, E_{z}, G_{x}, G_{z}$ are defined by Fig.1 and

$$
\begin{gathered}
\varepsilon_{x z}=E_{x} \cdot \sqrt{E_{z}^{2}-G_{x}^{2}}=E_{z} \cdot \sqrt{E_{x}^{2}-G_{z}^{2}} ; \\
J=\pi \cdot \varepsilon_{x z} ;
\end{gathered}
$$

( $\mathrm{J}$ is the area of the ellipse). 
3.) The charge density is a constant independent of position within the bunch. F.J.Sacherer has shown that in general for practical distributions, the dependence of the envelope equations on the type of distribution can be neglected [4]. However, since with a uniform distribution, the space charge varies linearly [2] with $\mathrm{x}$ and $\mathrm{z}$, the formalism can be greately simplified and the usual techniques of linear optics used (see section 3)[3].

4.) In calculating space charge forces we neglect the small spread in longitudinal momenta.

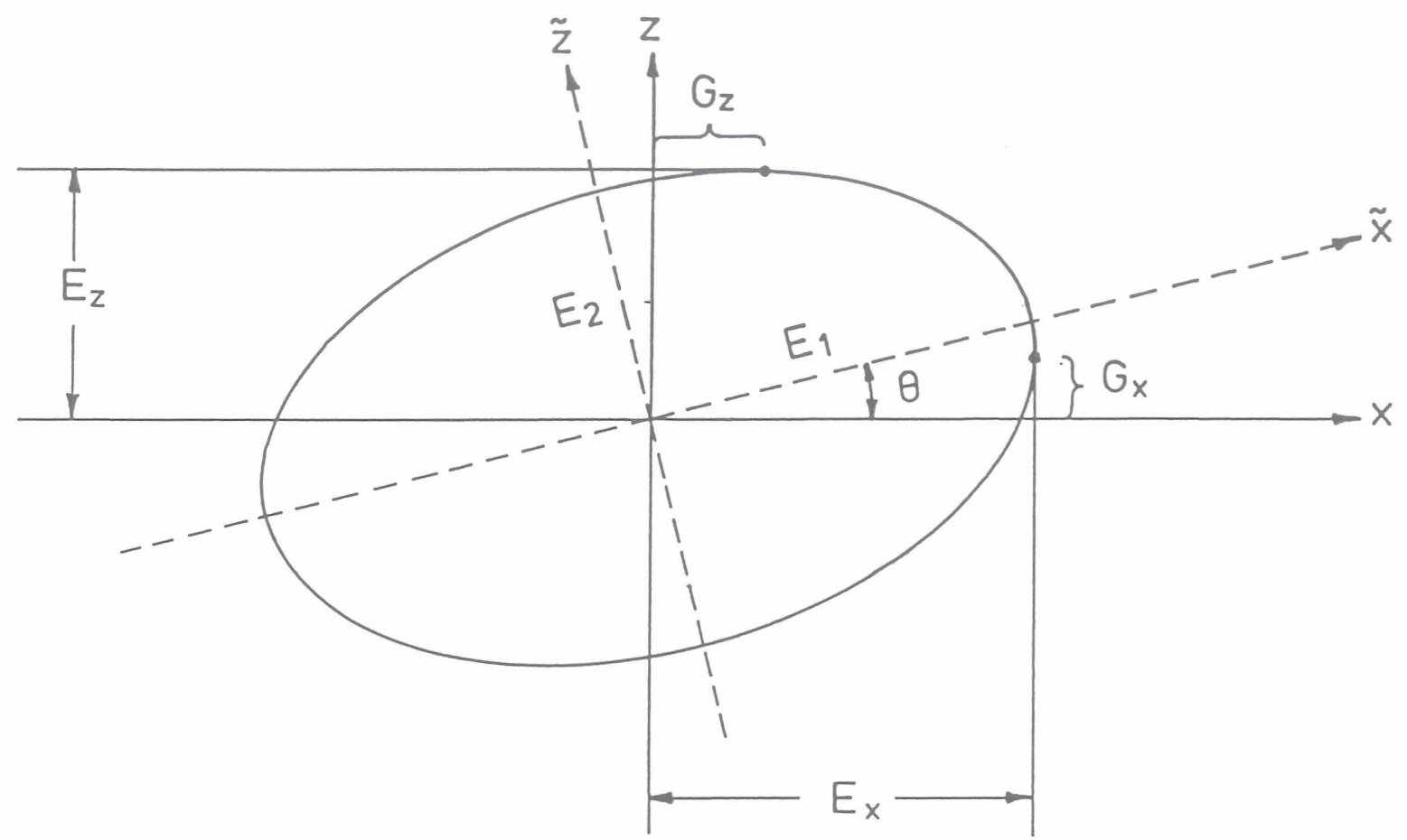

Fig. 1

With assumptions 1), 2), 3) and 4) and following J.M.Kapchinskij and V.V. Vladimirskij [2], in the rest system $\Sigma_{0}$ of the beam defined by $\Delta p / p=0$, the components of $\vec{F}^{\text {self }}$ along the symmetry axes $(\tilde{x}, \tilde{z})$ are given by $\left(E_{1}, E_{2}=\right.$ half axes of the ellipse) :

$$
\begin{aligned}
& F_{\tilde{x} 0}^{\text {self }}=e \cdot 4 \lambda_{0} \cdot \frac{1}{E_{1}\left(E_{1}+E_{2}\right)} \cdot \bar{x} ; \\
& F_{\tilde{z} 0}^{\text {self }}=e \cdot 4 \lambda_{0} \cdot \frac{1}{E_{2}\left(E_{1}+E_{2}\right)} \cdot \bar{z} ; \\
& F_{s 0}^{\text {self }}=0 ;
\end{aligned}
$$

$$
\left(\lambda_{0}=\text { line charge density in } \Sigma_{0}\right) .
$$

Then, with respect to the $(x, z)$ axes we obtain:

$$
F_{x 0}^{\text {self }}=F_{\tilde{x} 0}^{\text {self }} \cdot \cos \Theta-F_{\tilde{z} 0}^{\text {self }} \cdot \sin \Theta
$$




$$
F_{z 0}^{s e l f}=F_{\tilde{x} 0}^{\text {self }} \cdot \sin \Theta+F_{\tilde{z} 0}^{\text {self }} \cdot \cos \Theta
$$

Transforming into the lab. system $\Sigma$ we have:

$$
\begin{gathered}
F_{x}^{\text {self }}=\frac{1}{\gamma} \cdot F_{x 0}^{\text {self }} ; \\
F_{z}^{\text {self }}=\frac{1}{\gamma} \cdot F_{z 0}^{\text {self }} ; \\
F_{s}^{\text {self }}=0
\end{gathered}
$$

or, if we use the relation

$$
\lambda=\gamma \cdot \lambda_{0}
$$

(line charge density in $\Sigma$ )

(a calculation of $\lambda$ may be found in Ref.[5]) and substitute (2.5) and (2.6) in (2.7) :

$$
\begin{aligned}
& F_{x}^{\text {self }}=\frac{4 \lambda}{\gamma^{2}} \cdot e \cdot\left(F_{x x} \cdot x+F_{x z} \cdot z\right) \\
& F_{z}^{s e l f}=\frac{4 \lambda}{\gamma^{2}} \cdot e \cdot\left(F_{z x} \cdot x+F_{z z} \cdot z\right)
\end{aligned}
$$

with

$$
\begin{aligned}
& F_{x z}=F_{z x}=\frac{1}{\left(E_{1}+E_{2}\right)} \cdot\left(\frac{1}{E_{1}}-\frac{1}{E_{2}}\right) \cdot \sin \Theta \cos \Theta ; \\
& F_{x x}=\frac{1}{\left(E_{1}+E_{2}\right)} \cdot\left(\frac{1}{E_{1}} \cdot \cos ^{2} \Theta+\frac{1}{E_{2}} \cdot \sin ^{2} \Theta\right) \\
& F_{x z}=\frac{1}{\left(E_{1}+E_{2}\right)} \cdot\left(\frac{1}{E_{1}} \cdot \sin ^{2} \Theta+\frac{1}{E_{2}} \cdot \cos ^{2} \Theta\right)
\end{aligned}
$$

If the closed orbit is straight, equation (2.1), when written in terms of the $\mathrm{x}$-z-s components, takes the form $[7,8]$

$$
\begin{aligned}
\ddot{x} & =\frac{e}{m_{0} \gamma c} \cdot\left(\dot{z} B_{s}-\dot{s} B_{z}\right)+\frac{1}{m_{0} \gamma} \cdot F_{x}^{\text {self }} ; \\
\ddot{z} & =\frac{e}{m_{0} \gamma c} \cdot\left(\dot{s} B_{x}-\dot{x} B_{s}\right)+\frac{1}{m_{0} \gamma} \cdot F_{z}^{\text {self }} ; \\
\ddot{s} & =\frac{e}{m_{0} \gamma c} \cdot\left(\dot{x} B_{z}-\dot{z} B_{x}\right) .
\end{aligned}
$$

We now introduce $s$ as independent variable:

$$
\begin{array}{r}
\dot{x}=\frac{d x}{d s} \cdot \frac{d s}{d t}=x^{\prime} \cdot \dot{s} ; \quad \ddot{x}=x^{\prime \prime} \cdot \dot{s}^{2}+x^{\prime} \cdot \ddot{s} ; \\
\dot{z}=z^{\prime} \cdot \dot{s} ; \quad \ddot{z}=z^{\prime \prime} \cdot \dot{s}^{2}+z^{\prime} \cdot \ddot{s} ;
\end{array}
$$




$$
\begin{gathered}
v^{2}=\dot{s}^{2}+\dot{x}^{2}+\dot{z}^{2}=\dot{s}^{2} \cdot\left(1+x^{\prime 2}+z^{\prime 2}\right) ; \\
\frac{1}{\dot{s}}=\frac{1}{v} \sqrt{1+x^{\prime 2}+z^{\prime 2}}
\end{gathered}
$$

so that eqn.(2.11) becomes:

$$
\begin{aligned}
x^{\prime \prime}= & \frac{e}{p \cdot c} \sqrt{1+x^{\prime 2}+z^{\prime 2}} \cdot\left[z^{\prime} \cdot B_{s}-\left(1+x^{\prime 2}\right) \cdot B_{z}+x^{\prime} z^{\prime} \cdot B_{x}\right]+ \\
& \frac{\left(1+x^{\prime 2}+z^{\prime 2}\right)}{v^{2}} \cdot \frac{1}{m_{0} \gamma} \cdot F_{x}^{\text {self }} ; \\
z^{\prime \prime}= & -\frac{e}{p \cdot c} \sqrt{1+x^{\prime 2}+z^{\prime 2}} \cdot\left[x^{\prime} \cdot B_{s}-\left(1+z^{\prime 2}\right) \cdot B_{x}+x^{\prime} z^{\prime} \cdot B_{z}\right]+ \\
& \frac{\left(1+x^{\prime 2}+z^{\prime 2}\right)}{v^{2}} \cdot \frac{1}{m_{0} \gamma} \cdot F_{z}^{\text {self }} .
\end{aligned}
$$

Putting:

$$
\begin{aligned}
N & =\frac{1}{2} \cdot \frac{e}{p \cdot c}\left(\frac{\partial B_{x}}{\partial x}-\frac{\partial B_{z}}{\partial z}\right)_{x=z=0} \\
H & =\frac{1}{2} \cdot \frac{e}{p \cdot c} B_{s} \\
g & =\frac{e}{p \cdot c}\left(\frac{\partial B_{z}}{\partial x}\right)_{x=z=0}
\end{aligned}
$$

and using the relations:

$$
\begin{gathered}
\operatorname{div} \vec{B}=0 \Rightarrow \frac{\partial B_{x}}{\partial x}+\frac{\partial B_{z}}{\partial z}+\frac{\partial B_{s}}{\partial s}=0 ; \\
\operatorname{rot} \vec{B}=0 \Rightarrow \frac{\partial B_{z}}{\partial x}=\frac{\partial B_{x}}{\partial z},
\end{gathered}
$$

equation (2.14) in linearised form is then:

$$
\begin{aligned}
& x^{\prime \prime}+g \cdot x=\left(N+H^{\prime}\right) \cdot z+2 H \cdot z^{\prime}+\frac{1}{\gamma m_{0} v^{2}} \cdot F_{x}^{\text {self }} \\
& z^{\prime \prime}-g \cdot z=\left(N-H^{\prime}\right) \cdot x-2 H \cdot x^{\prime}+\frac{1}{\gamma m_{0} v^{2}} \cdot F_{z}^{\text {self }} .
\end{aligned}
$$

Equation (2.15) is valid for a straight design orbit. If on the contrary, the design orbit is curved in the horizontal plane, one must include an inhomogeneous term[6]

$$
+\frac{1}{\rho(s)} \cdot \frac{\Delta p}{p}
$$

on the r.h.s. of eqn. (2.15a), where $\rho(s)=$ curvature of the reference trajectory and $\Delta p$ is the deviation from the reference momentum $p_{0}$. Furthermore, the factor $\mathrm{g}$ is replaced by:

$$
g+\frac{1}{\rho^{2}} .
$$


The complete equations of motion (see (2.9)) including the effect of self fields and external guide fields are then:

$$
\begin{gathered}
x^{\prime \prime}+\left[g+\frac{1}{\rho^{2}}\right] \cdot x-\left(N+H^{\prime}\right) \cdot z-2 H \cdot z^{\prime}= \\
\frac{4 \lambda \cdot e}{\gamma^{3} \cdot m_{0} v^{2}} \cdot\left[F_{x x} \cdot x+F_{x z} \cdot z\right]+\frac{1}{\rho(s)} \cdot \frac{\Delta p}{p} ; \\
z^{\prime \prime}-g \cdot z-\left(N-H^{\prime}\right) \cdot x+2 H \cdot x^{\prime}= \\
\frac{4 \lambda \cdot e}{\gamma^{3} \cdot m_{0} v^{2}} \cdot\left[F_{z x} \cdot x+F_{z z} \cdot z\right] .
\end{gathered}
$$

In detail, one has:
a) $\quad g \neq 0 ; \quad N=H=\frac{1}{\rho}=0:$ quadrupole;
b) $N \neq 0 ; g=H=\frac{1}{\rho}=0$ : skew quadrupole;
c) $\frac{1}{\rho} \neq 0 ; g=N=H=0$ : bending magnet;
d) $H \neq 0 ; g=N=\frac{1}{\rho}=0:$ solenoid.

The term $F_{x z}=F_{z x}$ appearing in (2.16) describes the self-coupling of the bunch and vanishes for a cylindrical bunch according to eqn. (2.10) $\left(E_{1}=E_{2}\right)$.

For later developments, it is useful to introduce the momentum variables

$$
\begin{aligned}
& p_{x}=x^{\prime}-H \cdot z \\
& p_{z}=z^{\prime}+H \cdot x
\end{aligned}
$$

Equation (2.16) then becomes:

$$
\begin{gathered}
x^{\prime}=p_{x}+H \cdot z ; \\
p_{x}^{\prime}=-\left[g+\frac{1}{\rho^{2}}+H^{2}\right] \cdot x+N \cdot z+H \cdot p_{z}+\frac{4 \lambda \cdot e}{\gamma^{3} \cdot m_{0} v^{2}} \cdot\left[F_{x x} \cdot x+F_{x z} \cdot z\right]+\frac{1}{\rho(s)} \cdot \frac{\Delta p}{p} \\
z^{\prime}=p_{z}-H \cdot x \\
p_{z}^{\prime}=N \cdot x-H \cdot p_{x}+\left[g-H^{2}\right] \cdot z+\frac{4 \lambda \cdot e}{\gamma^{3} \cdot m_{0} v^{2}} \cdot\left[F_{z x} \cdot x+F_{z z} \cdot z\right]
\end{gathered}
$$

\section{$\underline{\text { Remark: }}$}

To handle particles with different energy deviations in a uniform manner we take for the constants $\gamma$ and $\mathrm{v}$ appearing in eqn. (2.18) the values belonging to $\Delta p / p=0$ (see eqn. (2.1)). 


\section{The Beam Envelopes}

\subsection{The 5-dimensional ellipsoid in $x-p_{x}-z-p_{z}-\Delta p / p$ space}

In obtaining equation (2.18), we have assumed that the beam cross section remains ellipse-like as the bunch travels along the closed orbit. This is indeed the case as we prove below but we must regard the $F_{x x}, F_{x z}, F_{z x}, F_{z z}$ as varying and as yet unknown functions of s. Equations (2.18) are then linear and their solutions can be expressed in the form

$$
\overrightarrow{\hat{y}}(s)=\underline{\hat{M}}\left(s, s_{0}\right) \overrightarrow{\hat{y}}\left(s_{0}\right)
$$

with

$$
\begin{gathered}
\overrightarrow{\hat{y}}=\left(\begin{array}{c}
\vec{y} \\
\Delta p / p
\end{array}\right) ; \quad \vec{y}=\left(\begin{array}{c}
x \\
p_{x} \\
z \\
p_{z}
\end{array}\right) ; \\
\underline{\hat{M}}\left(s, s_{0}\right)=\left(\begin{array}{cc}
\underline{M}\left(s, s_{0}\right) & \vec{x}\left(s, s_{0}\right) \\
0 & 1
\end{array}\right) .
\end{gathered}
$$

$\underline{\mathrm{M}}$ is the transfer matrix associated with the homogeneous part of eqn. $(2.18), \vec{x}$ is a special solution for the inhomogeneous system (2.18) with $\Delta p / p=1$ and for which

$$
\vec{x}\left(s_{0}, s_{0}\right)=0 .
$$

Since, according to $(2.10)$ we have

$$
F_{x z}(s)=F_{z x}(s),
$$

we can represent the equation of motion (2.18) in Hamiltonian form

$$
\begin{aligned}
& x^{\prime}=\frac{\partial \hat{H}}{\partial p_{x}} ; \quad p_{x}^{\prime}=-\frac{\partial \hat{H}}{\partial x} ; \\
& z^{\prime}=\frac{\partial \hat{H}}{\partial p_{z}} ; \quad p_{z}^{\prime}=-\frac{\partial \hat{H}}{\partial z}
\end{aligned}
$$

with the Hamiltonian[ $[7,8]$

$$
\begin{aligned}
\hat{H}= & \frac{1}{2} \cdot\left\{\left[g+\frac{1}{\rho^{2}}\right] \cdot x^{2}-g \cdot z^{2}-2 N \cdot x z+\left[p_{x}+H \cdot z\right]^{2}+\left[p_{z}-H \cdot x\right]^{2}\right\}- \\
& \frac{1}{2} \cdot \frac{4 \lambda \cdot e}{\gamma^{3} \cdot m_{0} v^{2}} \cdot\left[F_{x x} \cdot x^{2}+2 F_{x z} \cdot x z+F_{z z} \cdot z^{2}\right]- \\
& \frac{1}{\rho(s)} \cdot \frac{\Delta p}{p} \cdot x .
\end{aligned}
$$

Because the variables $x, p_{x}, z, p_{z}$ are canonical, the transfer matrix is symplectic [7,8]:

$$
\underline{M}^{T}\left(s, s_{0}\right) \cdot \underline{S} \cdot \underline{M}\left(s, s_{0}\right)=\underline{S}
$$


where

$$
\underline{S}=\left(\begin{array}{rrrr}
0 & -1 & 0 & 0 \\
1 & 0 & 0 & 0 \\
0 & 0 & 0 & -1 \\
0 & 0 & 1 & 0
\end{array}\right)
$$

As we prove later, we may ensure that the projections in the $x-z$ (and the $x-p_{x}$ and $z-p_{z}$ ) plane is elliptical by choosing a particle ensemble ( at $s=s_{0}$ ) which occupies a five dimensional ellipsoid in $x-p_{x}-z-p_{z}-\Delta p / p$ space whose surface is of the form[9]:

$$
\begin{aligned}
\overrightarrow{\hat{y}}\left(s_{0} ; \varphi, \chi, \delta_{I}, \delta_{I I}\right)= & \cos \varphi \cdot \cos \chi \cdot\left[\left(\begin{array}{c}
\vec{y}_{1}\left(s_{0}\right) \\
0
\end{array}\right) \cdot \cos \delta_{I}+\left(\begin{array}{c}
\vec{y}_{2}\left(s_{0}\right) \\
0
\end{array}\right) \cdot \sin \delta_{I}\right]+ \\
& \cos \varphi \cdot \sin \chi \cdot\left[\left(\begin{array}{c}
\vec{y}_{3}\left(s_{0}\right) \\
0
\end{array}\right) \cdot \cos \delta_{I I}+\left(\begin{array}{c}
\vec{y}_{4}\left(s_{0}\right) \\
0
\end{array}\right) \cdot \sin \delta_{I I}\right]+ \\
& \sin \varphi \cdot\left(\begin{array}{c}
\vec{y}_{5}\left(s_{0}\right) \\
(\Delta p / p)_{0}
\end{array}\right)
\end{aligned}
$$

The $\vec{y}_{i}\left(s_{0}\right)$ are five independent fixed orbit vectors:

$$
\begin{aligned}
&\left(\begin{array}{c}
\vec{y}_{k} \\
0
\end{array}\right)=\left(\begin{array}{c}
x_{k} \\
p_{x k} \\
z_{k} \\
p_{z k} \\
0
\end{array}\right) ; \quad(k=1,2,3,4) ; \\
&\left(\begin{array}{c}
\vec{y}_{5} \\
(\Delta p / p)_{0}
\end{array}\right)=\left(\begin{array}{c}
x_{5} \\
p_{x 5} \\
z_{5} \\
p_{z 5} \\
(\Delta p / p)_{0}
\end{array}\right)
\end{aligned}
$$

which are determined by the shape of the ellipsoid at $s_{0}$ and the surface is spanned by varying $\varphi, \chi, \delta_{I}, \delta_{I I}$.

We can see that eqn. (3.4) represents an ellipsoid as follows:

Eqn. (3.4) can also be written in the form:

$$
\overrightarrow{\hat{y}}=\left(\overrightarrow{\hat{y}_{1}}, \overrightarrow{\hat{y}_{2}}, \overrightarrow{\hat{y}_{3}}, \overrightarrow{\hat{y}_{4}}, \overrightarrow{\hat{y}_{5}}\right) \cdot \vec{q}
$$

with

$$
\begin{gathered}
\vec{q}=\left(\begin{array}{c}
\cos \varphi \cdot \cos \chi \cdot \cos \delta_{I} \\
\cos \varphi \cdot \cos \chi \cdot \sin \delta_{I} \\
\cos \varphi \cdot \sin \chi \cdot \cos \delta_{I I} \\
\cos \varphi \cdot \sin \chi \cdot \cos \delta_{I I} \\
\sin \varphi
\end{array}\right) ; \\
\overrightarrow{\hat{y}_{k}}=\left(\begin{array}{c}
\overrightarrow{y_{k}} \\
0
\end{array}\right) ;(k=1,2,3,4) ; \\
\overrightarrow{\hat{y}_{5}}=\left(\begin{array}{c}
\overrightarrow{y_{5}} \\
(\Delta p / p)_{0}
\end{array}\right) .
\end{gathered}
$$


It follows from (3.6) that

$$
\vec{q}=\underline{T} \cdot \overrightarrow{\hat{y}}
$$

where

$$
\underline{T}^{-1}=\left(\overrightarrow{\hat{y}_{1}}, \overrightarrow{\hat{y}_{2}}, \overrightarrow{\hat{y}_{3}}, \overrightarrow{\hat{y}_{4}}, \overrightarrow{\hat{y}_{5}}\right)
$$

(Note that the vectors $\overrightarrow{\hat{y}}_{k}(k=1,2,3,4,5)$ are linearly independent. Therefore the matrix $\left(\overrightarrow{\hat{y}}_{1}, \overrightarrow{\hat{y}}_{2}, \overrightarrow{\hat{y}}_{3}, \overrightarrow{\hat{y}}_{4}, \overrightarrow{\hat{y}}_{5}\right)$ is nonsingular and the inverse of this matrix exists.)

Furthermore, if we use the relation

$$
q_{1}^{2}+q_{2}^{2}+q_{3}^{2}+q_{4}^{2}+q_{5}^{2}=1
$$

we find that

$$
\sum_{\mu, \nu=1}^{5}\left[T_{\mu \nu} \cdot \hat{y}_{\nu}\right]^{2}=1 .
$$

Since the matrix $\mathrm{T}$ is nonsingular, the left hand side of eqn. (3.7) represents a positive definite quadratic form from which $\varphi, \chi, \delta_{I}, \delta_{I I}$ have been eliminated. This confirms that eqn. (3.4) indeed represents the surface of an ellipsoid in $x-p_{x}-z-p_{z}-\Delta p / p$ space.

During the particle motion, the eqn. (3.4) transforms to

$$
\begin{aligned}
& \overrightarrow{\hat{y}}\left(s ; \varphi, \chi, \delta_{I}, \delta_{I I}\right)=\underline{\hat{M}}\left(s, s_{0}\right) \overrightarrow{\hat{y}}\left(s_{0} ; \varphi, \chi, \delta_{I}, \delta_{I I}\right)= \\
& \cos \varphi \cdot \cos \chi \cdot\left[\left(\begin{array}{c}
\vec{y}_{1}(s) \\
0
\end{array}\right) \cdot \cos \delta_{I}+\left(\begin{array}{c}
\vec{y}_{2}(s) \\
0
\end{array}\right) \cdot \sin \delta_{I}\right]+ \\
& \quad \cos \varphi \cdot \sin \chi \cdot\left[\left(\begin{array}{c}
\vec{y}_{3}(s) \\
0
\end{array}\right) \cdot \cos \delta_{I I}+\left(\begin{array}{c}
\vec{y}_{4}(s) \\
0
\end{array}\right) \cdot \sin \delta_{I I}\right]+ \\
& \quad \sin \varphi \cdot\left(\begin{array}{c}
\vec{y}_{5}(s) \\
(\Delta p / p)_{0}
\end{array}\right)
\end{aligned}
$$

with

$$
\begin{array}{r}
\vec{y}_{k}(s)=\underline{M}\left(s, s_{0}\right) \vec{y}_{k}\left(s_{0}\right) ;(k=1,2,3,4) ; \\
\vec{y}_{5}(s)=\underline{M}\left(s, s_{0}\right) \vec{y}_{5}\left(s_{0}\right)+\left(\frac{\Delta p}{p}\right)_{0} \cdot \vec{x}\left(s, s_{0}\right),
\end{array}
$$

so that the beam envelope keeps its ellipsoidal form. It is thereby already clear that if the beam cross section, which is the projection of the ellipsoid (3.4) on the $x-z$ plane, is elliptical as required by (2.18), then the projection remains elliptical and can be described by eqn. (2.2) and (2.3).

In the next section we calculate the projections of the ellipsoid on the $x-p_{x}, z-p_{z}$ and $x-z$ planes.

\subsection{The projection of the five dimensional ellipsoid}

To calculate the projection and the equation of the ellipsoid (3.8) we first of all express eqn. (3.8) in component form:

$$
x\left(s ; \varphi, \chi, \delta_{I}, \delta_{I I}\right)=\cos \varphi \cdot \cos \chi \cdot\left[y_{11}(s) \cdot \cos \delta_{I}+y_{21}(s) \cdot \sin \delta_{I}\right]+
$$




$$
\begin{aligned}
& \cos \varphi \cdot \sin \chi \cdot\left[y_{31}(s) \cdot \cos \delta_{I I}+y_{41}(s) \cdot \sin \delta_{I I}\right]+ \\
& \sin \varphi \cdot y_{51}(s) ; \\
p_{x}\left(s ; \varphi, \chi, \delta_{I}, \delta_{I I}\right)= & \cos \varphi \cdot \cos \chi \cdot\left[y_{12}(s) \cdot \cos \delta_{I}+y_{22}(s) \cdot \sin \delta_{I}\right]+ \\
& \cos \varphi \cdot \sin \chi \cdot\left[y_{32}(s) \cdot \cos \delta_{I I}+y_{42}(s) \cdot \sin \delta_{I I}\right]+ \\
& \sin \varphi \cdot y_{52}(s) ; \\
z\left(s ; \varphi, \chi, \delta_{I}, \delta_{I I}\right)= & \cos \varphi \cdot \cos \chi \cdot\left[y_{13}(s) \cdot \cos \delta_{I}+y_{23}(s) \cdot \sin \delta_{I}\right]+ \\
& \cos \varphi \cdot \sin \chi \cdot\left[y_{33}(s) \cdot \cos \delta_{I I}+y_{43}(s) \cdot \sin \delta_{I I}\right]+ \\
& \sin \varphi \cdot y_{53}(s) ; \\
p_{z}\left(s ; \varphi, \chi, \delta_{I}, \delta_{I I}\right)= & \cos \varphi \cdot \cos \chi \cdot\left[y_{14}(s) \cdot \cos \delta_{I}+y_{24}(s) \cdot \sin \delta_{I}\right]+ \\
& \cos \varphi \cdot \sin \chi \cdot\left[y_{34}(s) \cdot \cos \delta_{I I}+y_{44}(s) \cdot \sin \delta_{I I}\right]+ \\
& \sin \varphi \cdot y_{54}(s) .
\end{aligned}
$$

The projections are then obtained by investigating the functional dependences of pairs of components[9].

\subsubsection{Projection on the $x-z$ plane.}

The projection on the $x-z$ plane describes the boundary of the beam cross section. Thus it is useful to calculate the maximum amplitudes in the two directions.

a) Maximum oscillation amplitude in $x$ direction:

By using the relation

$$
\operatorname{Max}_{\varphi}\{A \cdot \cos \varphi+B \cdot \sin \varphi\}=\sqrt{A^{2}+B^{2}}
$$

we see from $(3.10 a)$ that the maximum amplitude in the $\mathrm{x}$ direction is

$$
\operatorname{Max}_{\left(\varphi, \chi, \delta_{I}, \delta_{I I}\right)} x\left(s ; \varphi, \chi, \delta_{I}, \delta_{I I}\right)=\sqrt{y_{11}^{2}+y_{21}^{2}+y_{31}^{2}+y_{41}^{2}+y_{51}^{2}} .
$$

If we anticipate that the projection is an ellipse, the maximum amplitude in (3.11a) can be identified with the ellipse envelope parameter of eqn. (2.2), i.e.

$$
E_{x}(s)=\sqrt{y_{11}^{2}+y_{21}^{2}+y_{31}^{2}+y_{41}^{2}+y_{51}^{2}} .
$$

This amplitude is reached for the case where the angles $\delta_{I}, \delta_{I I}, \chi, \varphi$ are given by

$$
\begin{gathered}
\cos \delta_{I}=\frac{y_{11}}{\sqrt{y_{11}^{2}+y_{21}^{2}}} ; \quad \sin \delta_{I}=\frac{y_{21}}{\sqrt{y_{11}^{2}+y_{21}^{2}}} ; \\
\cos \delta_{I I}=\frac{y_{31}}{\sqrt{y_{31}^{2}+y_{41}^{2}}} ; \quad \sin \delta_{I I}=\frac{y_{41}}{\sqrt{y_{31}^{2}+y_{41}^{2}}} ; \\
\cos \chi=\frac{\sqrt{y_{11}^{2}+y_{21}^{2}}}{\sqrt{y_{11}^{2}+y_{21}^{2}+y_{31}^{2}+y_{41}^{2}}} ; \\
\sin \chi=\frac{\sqrt{y_{31}^{2}+y_{41}^{2}}}{\sqrt{y_{11}^{2}+y_{21}^{2}+y_{31}^{2}+y_{41}^{2}}} ;
\end{gathered}
$$




$$
\begin{aligned}
& \cos \varphi=\frac{\sqrt{y_{11}^{2}+y_{21}^{2}+y_{31}^{2}+y_{41}^{2}}}{\sqrt{y_{11}^{2}+y_{21}^{2}+y_{31}^{2}+y_{41}^{2}+y_{51}^{2}}} ; \\
& \sin \varphi=\frac{y_{51}}{\sqrt{y_{11}^{2}+y_{21}^{2}+y_{31}^{2}+y_{41}^{2}+y_{51}^{2}}} .
\end{aligned}
$$

The corresponding $\mathrm{z}$ coordinate can then be obtained from eqn. (3.10c):

$$
G_{x}=\frac{1}{E_{x}(s)} \cdot\left\{y_{11} \cdot y_{13}+y_{21} \cdot y_{23}+y_{31} \cdot y_{33}+y_{41} \cdot y_{43}+y_{51} \cdot y_{53}\right\} .
$$

b) Maximum oscillation amplitude in $z$ direction:

The maximum amplitude in $\mathrm{z}$ direction is calculated in the same way. From eqn. (3.10c):

$$
\operatorname{Max}_{\left(\varphi, \chi, \delta_{I}, \delta_{I I}\right)} z\left(s ; \varphi, \chi, \delta_{I}, \delta_{I I}\right)=\sqrt{y_{13}^{2}+y_{23}^{2}+y_{33}^{2}+y_{43}^{2}+y_{53}^{2}}=E_{z}(s) .
$$

The corresponding $\mathrm{x}$ coordinate is then

$$
G_{z}=\frac{1}{E_{z}(s)} \cdot\left\{y_{11} \cdot y_{13}+y_{21} \cdot y_{23}+y_{31} \cdot y_{33}+y_{41} \cdot y_{43}+y_{51} \cdot y_{53}\right\}
$$

Thus we see

$$
E_{x} \cdot G_{x}=E_{z} \cdot G_{z}
$$

c) Maximum oscillation amplitude at the angle $\vartheta$ with the $\mathrm{x}$ axis.

To calculate the equation of the beam cross section we now consider the largest amplitude that can occur along some direction which makes an angle $\vartheta$ with the $\mathrm{x}$ axis in the $x-z$ plane. It is useful to introduce a rotated coordinate system $\hat{x}-\hat{z}$ according to the transformation

$$
\begin{aligned}
& \hat{x}(\vartheta)=x \cdot \cos \vartheta+z \cdot \sin \vartheta ; \\
& \hat{z}(\vartheta)=-x \cdot \sin \vartheta+z \cdot \cos \vartheta .
\end{aligned}
$$

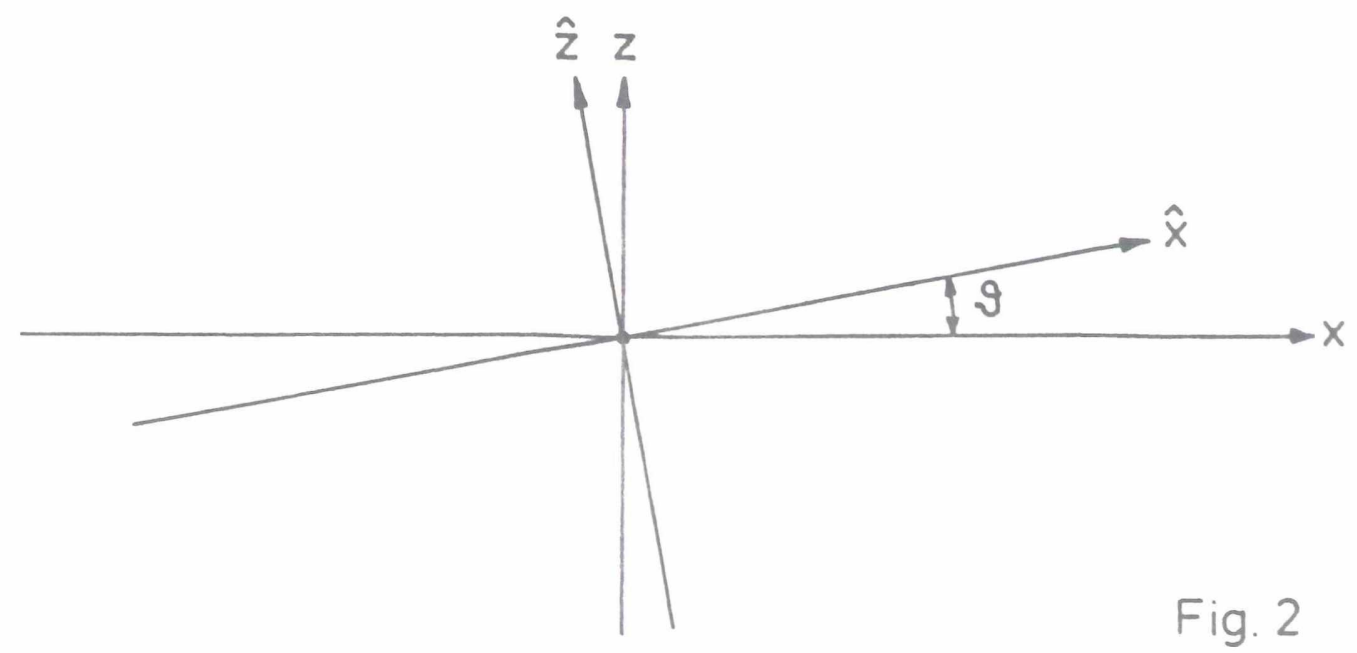

From eqns. (3.10a) and (3.10c)

$\hat{x}(\vartheta)=\cos \varphi \cos \chi\left[\left(y_{11} \cdot \cos \vartheta+y_{13} \cdot \sin \vartheta\right) \cos \delta_{I}+\left(y_{21} \cdot \cos \vartheta+y_{23} \cdot \sin \vartheta\right) \sin \delta_{I}\right]+$ 


$$
\begin{aligned}
& \cos \varphi \sin \chi\left[\left(y_{31} \cdot \cos \vartheta+y_{33} \cdot \sin \vartheta\right) \cos \delta_{I I}+\left(y_{41} \cdot \cos \vartheta+y_{43} \cdot \sin \vartheta\right) \sin \delta_{I I}\right]+ \\
& \sin \varphi \cdot\left(y_{51} \cdot \cos \vartheta+y_{53} \cdot \sin \vartheta\right)
\end{aligned}
$$

and for the maximum amplitude in the rotated frame

$$
\operatorname{Max}_{\left(\varphi, \chi, \delta_{I}, \delta_{I I}\right)} \hat{x}(\vartheta)=E(\vartheta)
$$

in the $\vartheta$ direction one obtains

$$
E(\vartheta)=\left\{\frac{1}{2}\left[E_{x}^{2}-E_{z}^{2}\right] \cdot \cos 2 \vartheta+E_{x} G_{x} \cdot \sin 2 \vartheta+\frac{1}{2}\left[E_{x}^{2}+E_{z}^{2}\right]\right\}^{1 / 2} .
$$

This amplitude is reached for the case where the angles $\delta_{I}, \delta_{I I}, \chi, \varphi$ are given by

$$
\begin{gathered}
\cos \delta_{I}=\frac{\xi_{1}(\vartheta)}{\sqrt{\xi_{1}^{2}+\xi_{2}^{2}}} ; \sin \delta_{I}=\frac{\xi_{2}(\vartheta)}{\sqrt{\xi_{1}^{2}+\xi_{2}^{2}}} ; \\
\cos \delta_{I I}=\frac{\xi_{3}(\vartheta)}{\sqrt{\xi_{3}^{2}+\xi_{4}^{2}}} ; \sin \delta_{I I}=\frac{\xi_{4}(\vartheta)}{\sqrt{\xi_{3}^{2}+\xi_{4}^{2}}} ; \\
\cos \chi=\frac{\sqrt{\xi_{1}^{2}+\xi_{2}^{2}}}{\sqrt{\xi_{1}^{2}+\xi_{2}^{2}+\xi_{3}^{2}+\xi_{4}^{2}}} ; \\
\sin \chi=\frac{\sqrt{\xi_{3}^{2}+\xi_{4}^{2}}}{\sqrt{\xi_{1}^{2}+\xi_{2}^{2}+\xi_{3}^{2}+\xi_{4}^{2}}} \\
\cos \varphi=\frac{\sqrt{\xi_{1}^{2}+\xi_{2}^{2}+\xi_{3}^{2}+\xi_{4}^{2}}}{\sqrt{\xi_{1}^{2}+\xi_{2}^{2}+\xi_{3}^{2}+\xi_{4}^{2}+\xi_{5}^{2}}} ; \\
\sin \varphi=\frac{\xi_{5}}{\sqrt{\xi_{1}^{2}+\xi_{2}^{2}+\xi_{3}^{2}+\xi_{4}^{2}+\xi_{5}^{2}}} .
\end{gathered}
$$

with

$$
\begin{aligned}
& \xi_{1}(\vartheta)=y_{11} \cdot \cos \vartheta+y_{13} \cdot \sin \vartheta ; \\
& \xi_{2}(\vartheta)=y_{21} \cdot \cos \vartheta+y_{23} \cdot \sin \vartheta ; \\
& \xi_{3}(\vartheta)=y_{31} \cdot \cos \vartheta+y_{33} \cdot \sin \vartheta ; \\
& \xi_{4}(\vartheta)=y_{41} \cdot \cos \vartheta+y_{43} \cdot \sin \vartheta ; \\
& \xi_{5}(\vartheta)=y_{51} \cdot \cos \vartheta+y_{53} \cdot \sin \vartheta .
\end{aligned}
$$

The angles $\vartheta_{1}$ and $\vartheta_{2}$ corresponding to the extrema of $E(\vartheta)$ are given by

$$
\begin{aligned}
0=\frac{d}{d \vartheta} E^{2}(\vartheta) & =-\left[E_{x}^{2}-E_{z}^{2}\right] \cdot \sin 2 \vartheta+2 E_{x} G_{x} \cdot \cos 2 \vartheta \\
\text { for } \vartheta & =\vartheta_{1,2}
\end{aligned}
$$


whence

$$
\begin{array}{r}
\tan 2 \vartheta_{1,2}=\frac{2 E_{x} G_{x}}{E_{x}^{2}-E_{z}^{2}} \\
\cos 2 \vartheta_{1,2}=\frac{E_{x}^{2}-E_{z}^{2}}{ \pm \sqrt{\left[E_{x}^{2}-E_{z}^{2}\right]^{2}+4 E_{x}^{2} G_{x}^{2}}}
\end{array}
$$

corresponding to extrema

$$
E_{1,2}^{2}=\frac{1}{2} \cdot\left\{\left[E_{x}^{2}-E_{z}^{2}\right] \pm \sqrt{\left[E_{x}^{2}-E_{z}^{2}\right]^{2}+4 E_{x}^{2} G_{x}^{2}}\right\}
$$

From (3.18b) we see that the directions corresponding to the angles $\vartheta_{1}$ and $\vartheta_{2}$ are perpendicular as one expects if the $x-z$ projection is elliptical.

Eqn. (3.17) describes the envelope of the beam in the $\vartheta$ direction. In particular for $\vartheta=0$

$$
E(0)=E_{x}
$$

and for $\vartheta=\pi / 2$

$$
E(\pi / 2)=E_{z}
$$

The $\hat{z}$ component corresponding to the $\hat{x}$ component $E(\vartheta)$ of eqn. (3.17) is given by

$$
G(\vartheta)=\frac{1}{E(\vartheta)} \cdot\left\{-\frac{1}{2}\left[E_{x}^{2}-E_{z}^{2}\right] \cdot \sin 2 \vartheta+E_{x} G_{x} \cdot \cos 2 \vartheta\right\}
$$

Special cases of eqn.(3.19) are:

$$
G(0)=G_{x}
$$

for $\vartheta=0$ and

$$
G(\pi / 2)=G_{z}
$$

for $\vartheta=\pi / 2($ see eqn.(3.16)).

d) The boundary curve of the beam cross section.

We will now show that the projection of the ellipsoid onto the $x-z$ plane is indeed elliptical. To achieve this, consider the ellipse:

$$
\left(\begin{array}{c}
x(s ; \psi) \\
z(s ; \psi)
\end{array}\right)=\left(\begin{array}{c}
E_{x}(s) \\
G_{x}(s)
\end{array}\right) \cdot \cos \psi+\left(\begin{array}{c}
0 \\
\sqrt{E_{z}^{2}-G_{x}^{2}}
\end{array}\right) \cdot \sin \psi
$$

where $\psi$ is a free parameter and where $E_{x}(s), G_{x}(s)$ and $E_{z}(s)$ are given by eqns. (3.11), (3.13) and (3.14). We see that if one takes the projection of the ellipse of (3.20) onto the $\hat{x}$ and $\hat{z}$ axes for each $\vartheta$ (Fig. 2) one just obtains the expression $E(\vartheta)$ of eqn. (3.17) and $G(\vartheta)$ of eqn. (3.19). Thus the ellipse (3.20) is identical to the boundary of the beam cross section in the $x-z$ plane.

Furthermore by eliminating $\cos \psi$ and $\sin \psi$ one obtains the ellipse of eqns. (2.2) and (2.3) which was used in Chapter 2 to calculate the space charge forces. 


\subsubsection{Projection on the $x-p_{x}$ plane.}

For the projection of the ellipsoid (3.8) onto the $x-p_{x}$ plane the corresponding equations are $(3.10 a),(3.10 b)$. Since these two relations have the same form as eqns. (3.10a) and (3.10c), we obtain an elliptical projection onto the $x-p_{x}$ plane by analogy with eqns. (2.2), (2.3). We write the ellipse in the form:

$$
A_{x}^{2} \cdot x^{2}-2 E_{x} E_{p_{x}} \cdot x p_{x}+E_{x}^{2} \cdot p_{x}^{2}=\varepsilon_{x}^{2}
$$

with

$$
\begin{gathered}
A_{x}(s)=\operatorname{Max}_{\left(\varphi, \chi, \delta_{I}, \delta_{2}\right)} p_{x}\left(s, \varphi, \chi, \delta_{I}, \delta_{I I}\right)=\sqrt{y_{12}^{2}+y_{22}^{2}+y_{32}^{2}+y_{42}^{2}+y_{52}^{2}} \\
E_{p_{x}}(s)=\frac{1}{E_{x}(s)} \cdot\left\{y_{11} \cdot y_{12}+y_{21} \cdot y_{22}+y_{31} \cdot y_{32}+y_{41} \cdot y_{42}+y_{51} \cdot y_{52}\right\} \\
\pi \varepsilon_{x}=\pi \cdot E_{x} \sqrt{A_{x}^{2}-E_{p_{x}}^{2}} \\
\text { (area of the ellipse }(3.21)) .
\end{gathered}
$$

Here, the function $A_{x}(s)$ represents the maximum amplitude of the momentum $p_{x}$ and could be called the momentum envelope for the $x-p_{x}$ plane. $\pi \varepsilon_{x}$ gives the area of the ellipse (3.18) and the meaning of $E_{p_{x}}$ is indicated in Fig. 3.

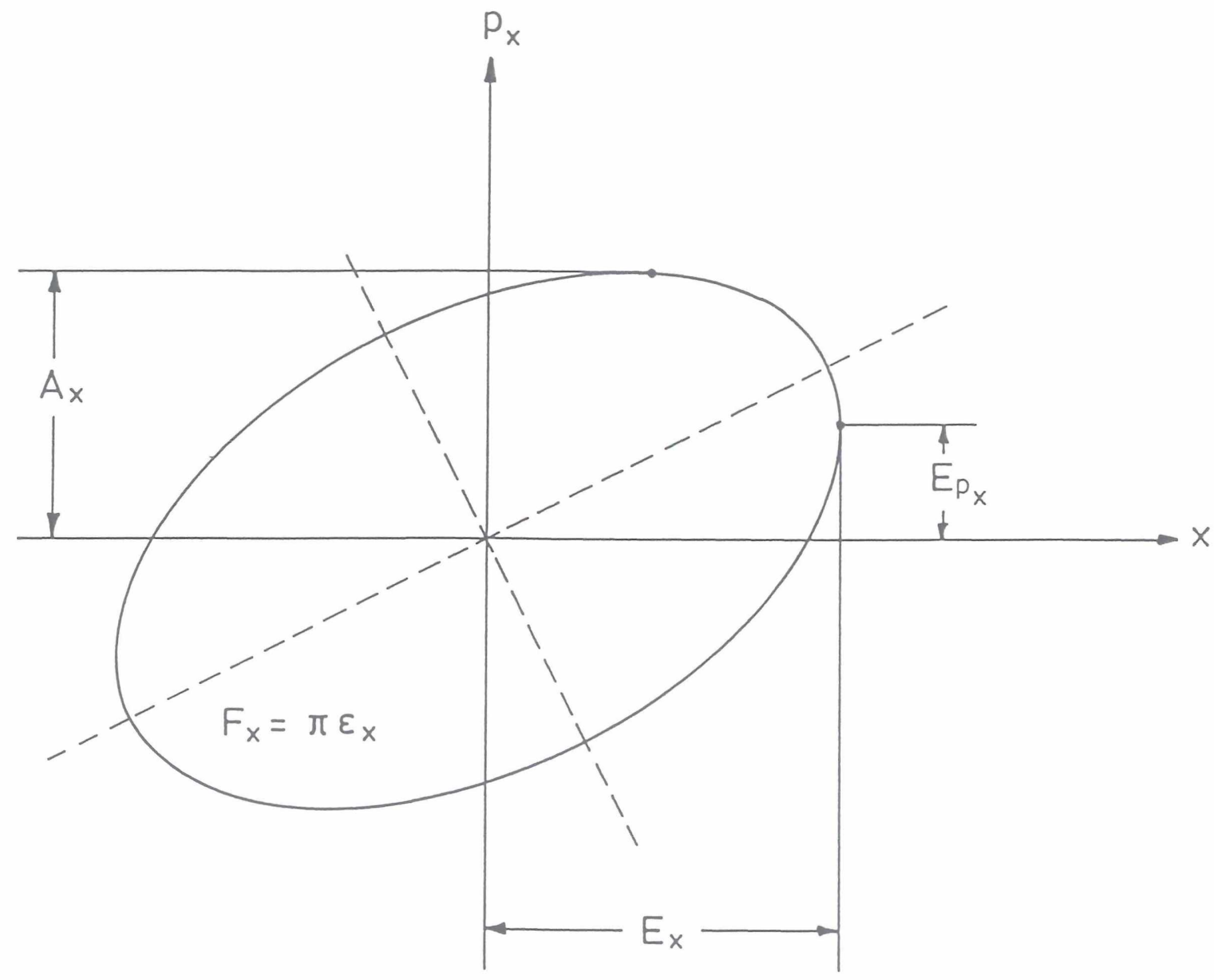

Fig. 3 


\subsubsection{Projection on the $z-p_{z}$ plane.}

A similar treatment can be used to describe the projection on the $z-p_{z}$ plane. We write

$$
A_{z}^{2} \cdot z^{2}-2 E_{z} E_{p_{z}} \cdot z p_{z}+E_{z}^{2} \cdot p_{z}^{2}=\varepsilon_{z}^{2}
$$

where

$$
\begin{gathered}
A_{z}(s)=\operatorname{Max}_{\left(\varphi, \chi, \delta_{I}, \delta_{I I}\right)} p_{z}\left(s, \varphi, \chi, \delta_{I}, \delta_{I I}\right)=\sqrt{y_{14}^{2}+y_{24}^{2}+y_{34}^{2}+y_{44}^{2}+y_{54}^{2}} \\
E_{p_{z}}(s)=\frac{1}{E_{z}(s)} \cdot\left\{y_{13} \cdot y_{14}+y_{23} \cdot y_{24}+y_{33} \cdot y_{34}+y_{43} \cdot y_{44}+y_{53} \cdot y_{54}\right\} \\
\pi \varepsilon_{z}=\pi \cdot E_{z} \sqrt{A_{z}^{2}-E_{p_{z}}^{2}} \\
\text { (area of the ellipse(3.25).) }
\end{gathered}
$$

This is all represented by the ellipse in Fig. 4.

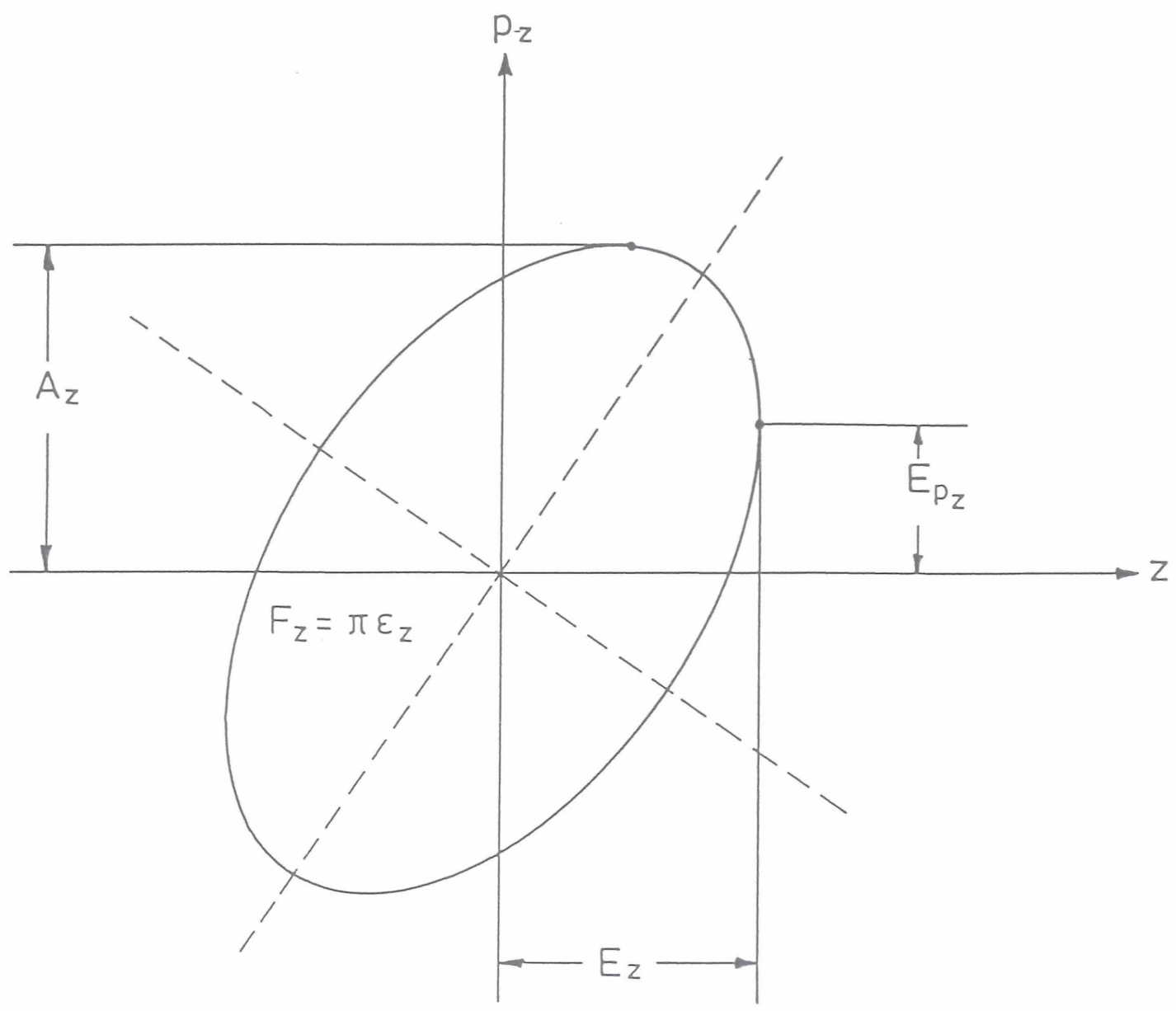

Fig. 4

With the calculation of the beam and momentum envelopes and with the proof that the beam cross section for general particle motion can be described by the ellipse of eqn. (2.2) we are now ready to return to the discussion of space charge forces of Chapter 2. 
We point out finally that the angle $\Theta$ of Fig.1 is to be identified with the angle $\vartheta_{1}$ of eqns. $(3.18 \mathrm{a}, \mathrm{b})$ and that the quantities $E_{1}, E_{2}$ appearing in eqns. (2.5), (2.10) and (2.18) are given by eqn. (3.18c).

A method of approximate solution of the equations of motion (2.16) will be presented in the next section.

\section{Remark:}

If we neglect the momentum spread, the 5-dimensional ellipsoid becomes a 4-dimensional ellipsoid :

$$
\begin{aligned}
\overrightarrow{\hat{y}}\left(s ; \chi, \delta_{I}, \delta_{I I}\right)= & \cos \chi \cdot\left[\vec{y}_{1}(s) \cdot \cos \delta_{I}+\vec{y}_{2}(s) \cdot \sin \delta_{I}\right]+ \\
& \sin \chi \cdot\left[\vec{y}_{3}(s) \cdot \cos \delta_{I I}+\vec{y}_{4}(s) \cdot \sin \delta_{I I}\right] .
\end{aligned}
$$

In this case the terms $E_{x}, E_{z}, E_{p_{x}}, E_{p_{z}}, A_{x}, A_{z}, G_{x}, G_{z}, \varepsilon_{x}, \varepsilon_{z}$ are obtained from eqns. (3.11), (3.15), (3.22), (3.24), (3.26 - 28) by setting $y_{5 \nu}=0$. The projections onto the $x-z, x-p_{x}$ and $z-p_{z}$ plane are again given by eqns. (2.2), (2.3), (3.21) and (3.25).

\section{Solution of the Equations of Motion}

\subsection{Thin lens approximation}

In matrix form, the equations of motion (2.18) are:

$$
\frac{d}{d s} \vec{y}=\underline{A}(s) \cdot \vec{y}+\frac{\Delta p}{p} \cdot \vec{r}
$$

with

$$
\begin{aligned}
& A_{11}=0 ; \\
& A_{12}=1 ; \\
& A_{13}=H ; \\
& A_{14}=0 ; \\
& A_{21}=-\left[g+\frac{1}{\rho^{2}}+H^{2}\right]+\frac{4 \lambda \cdot e}{\gamma^{3} \cdot m_{0} v^{2}} \cdot F_{x x} ; \\
& A_{22}=0 ; \\
& A_{23}=N+\frac{4 \lambda \cdot e}{\gamma^{3} \cdot m_{0} v^{2}} \cdot F_{x z} ; \\
& A_{24}=+H ; \\
& A_{31}=-H ; \\
& A_{32}=0 ; \\
& A_{33}=0 ; \\
& A_{34}=1 ; \\
& A_{41}=N+\frac{4 \lambda \cdot e}{\gamma^{3} \cdot m_{0} v^{2}} \cdot F_{z x} ;
\end{aligned}
$$




$$
\begin{aligned}
& A_{42}=-H ; \\
& A_{43}=\left[g-H^{2}\right]+\frac{4 \lambda \cdot e}{\gamma^{3} \cdot m_{0} v^{2}} \cdot F_{z z} ; \\
& A_{44}=0
\end{aligned}
$$

and

$$
\vec{r}=\left(\begin{array}{c}
0 \\
1 / \rho \\
0 \\
0
\end{array}\right)
$$

or, by using the vector $\overrightarrow{\hat{y}}$ of eqn. (3.1b):

$$
\frac{d}{d s} \overrightarrow{\hat{y}}=\left(\begin{array}{cc}
\underline{A} & \vec{r} \\
0 & 0
\end{array}\right) \overrightarrow{\hat{y}}
$$

For the transfer matrix $\underline{\hat{M}}\left(s, s_{0}\right)$ defined in eqn. (3.1c) we obtain the defining equation:

$$
\begin{aligned}
\frac{d}{d s} \underline{\hat{M}}\left(s, s_{0}\right) & =\left(\begin{array}{cc}
\underline{A}(s) & \vec{r} \\
0 & 0
\end{array}\right) \underline{\hat{M}}\left(s, s_{0}\right) ; \\
\underline{\hat{M}}\left(s_{0}, s_{0}\right) & =\underline{1}
\end{aligned}
$$

which in terms of components $\underline{M}\left(s, s_{0}\right)$ and $\vec{x}\left(s, s_{0}\right)$ gives:

$$
\begin{aligned}
& \frac{d}{d s} \vec{x}\left(s, s_{0}\right)=\underline{A}(s) \cdot \vec{x}\left(s, s_{0}\right)+\vec{r} ; \vec{x}\left(s_{0}, s_{0}\right)=0 ; \\
& \frac{d}{d s} \underline{M}\left(s, s_{0}\right)=\underline{A}(s) \cdot \underline{M}\left(s, s_{0}\right) ; \underline{M}\left(s_{0}, s_{0}\right)=\underline{1},
\end{aligned}
$$

so that in first approximation we may write :

$$
\Rightarrow \underline{M}(s+\Delta s, s)=\underline{1}+\Delta s \cdot \underline{A}(s)
$$

However, $\underline{M}$ so calculated is not symplectic (see eqn. (2.3)) for finite $\Delta s$.

The solution of eqn. (4.4), and also of eqn. (4.1), can be obtained however in a thin lens approximation which is also symplectic.

For this, we divide a lens into a sufficient number of thin lenses for which in the power series expansion of the matrix $\underline{\hat{M}}\left(s+\Delta s, s_{0}\right)$, only the linear terms are needed. Thus we write

$$
\hat{\underline{M}}(s+\Delta s, s)=\left(\begin{array}{cc}
\underline{M}(s+\Delta s, s) & \vec{r} \cdot \Delta s \\
\underline{0} & 1
\end{array}\right),
$$

for which $\underline{M}(s+\Delta s, s)$ is given approximately by eqn. (4.6).

To ensure that the symplecticity of the submatrix $\underline{M}(s+\Delta s, s)$ is rigorously maintained by the linearisation ( see eqn. (3.3)) we write

$$
\underline{M}(s+\Delta s, s)=\underline{M}_{D}\left(s+\Delta s, s+\frac{\Delta s}{2}\right) \cdot[\underline{1}+\underline{C}(s) \cdot \Delta s] \cdot \underline{R}(\Delta \Theta) \cdot \underline{M}_{D}\left(s+\frac{\Delta s}{2}, s\right)
$$


with

$$
\begin{gathered}
\underline{C}(s)=\underline{A}(s)-\underline{D}-\underline{F} ; \\
\underline{D}=\left(\begin{array}{rrrr}
0 & 1 & 0 & 0 \\
0 & 0 & 0 & 0 \\
0 & 0 & 0 & 1 \\
0 & 0 & 0 & 0
\end{array}\right) ; \\
\underline{F}=H \cdot\left(\begin{array}{rrrr}
0 & 0 & 1 & 0 \\
0 & 0 & 0 & 1 \\
-1 & 0 & 0 & 0 \\
0 & -1 & 0 & 0
\end{array}\right) ; \\
\underline{M}_{D}(s+l, s)=\underline{1}+l \cdot \underline{D} ;
\end{gathered}
$$

(transfer matrix for a simple drift space of length 1);

$$
\begin{gathered}
\underline{R}(\Delta \Theta)=\left(\begin{array}{rrrr}
\cos \Delta \Theta & 0 & +\sin \Delta \Theta & 0 \\
0 & \cos \Delta \Theta & 0 & +\sin \Delta \Theta \\
-\sin \Delta \Theta & 0 & \cos \Delta \Theta & 0 \\
0 & -\sin \Delta \Theta & 0 & \cos \Delta \Theta
\end{array}\right) ; \\
(\Delta \Theta=H \cdot \Delta s) .
\end{gathered}
$$

In linear order, the right hand side of (4.8) agrees with the r.h.s. of eqn. (4.6). Furthermore, all factor matrices on the r.h.s. of (4.8) and therefore $\underline{M}(s+\Delta s, s)$ itself are symplectic.

In this way, the linear approximation (4.6) for $\underline{M}(s+\Delta s, s)$ can be made symplectic by adding terms of higher order in $\Delta s$.

To compute the matrix $\hat{\underline{M}}(s+\Delta s, s)$ using eqn. (4.7) and (4.8) the coefficient matrix, $\underline{A}(s)$, must be known. But (eqn. (4.2a)), the matrix elements $A_{21}, A_{23}, A_{41}, A_{43}$ contain the quantities $F_{x x}, F_{x z}, F_{z x}$ and $F_{z z}$ and these depend on the space charge forces. Also, according to $(2.10),(3.19),(3.11),(3.13)$ and $(3.14)$ the $F^{\prime s}$ depend on the generating orbit vectors $\vec{y}_{k}$ $(k=1,2,3,4,5)$ whose behaviour also of course depends on the shape of the beam.

However, one should note that the vectors $\vec{y}_{k}$ can be calculated using the transfer matrix $\hat{\underline{M}}(s+\Delta s, s)$ once the starting conditions are known. By eqn. (3.9):

$$
\begin{aligned}
&\left(\begin{array}{c}
\vec{y}_{k}(s+\Delta s) \\
0
\end{array}\right)=\underline{\hat{M}}(s+\Delta s, s)\left(\begin{array}{c}
\vec{y}_{k}(s) \\
0
\end{array}\right) \\
& \text { for } \mathrm{k}=1,2,3,4 ; \\
&\left(\begin{array}{c}
\vec{y}_{5}(s+\Delta s) \\
(\Delta p / p)_{0}
\end{array}\right)=\underline{\hat{M}}(s+\Delta s, s)\left(\begin{array}{c}
\vec{y}_{5}(s) \\
(\Delta p / p)_{0}
\end{array}\right) .
\end{aligned}
$$


By using this relation together with (4.7), (4.8), (4.2a,b), (2.10), (3.18) as well as (3.11), (3.13),(3.14), (3.16) the transfer matrix for a thin slice can be defined and then, by multiplication, the matrix for a thick lens.

Finally we mention, that eqns. (4.10a,b) can be written in a compact form

$$
\underline{B}(s+\Delta s, s)=\underline{\hat{M}}(s+\Delta s, s) \cdot \underline{B}(s)
$$

by introducing the "bunch-shape matrix"

$$
\underline{B}=\left(\overrightarrow{\hat{y}_{1}}, \overrightarrow{\hat{y}_{2}}, \overrightarrow{\hat{y}_{3}}, \overrightarrow{\hat{y}_{4}}, \overrightarrow{\hat{y}_{5}}\right)
$$

which already appears in eqn. (3.6). This matrix $\underline{B}$ contains complete information about the configuration of the bunch - see eqns. (2.2), (3.21) and (3.25).

\subsection{Initial conditions for the ellipsoid}

The beam cross section depends both on the shape of the phase ellipsoid at injection and on the space charge forces. Thus, for example, in a machine with uncoupled optics, it is possible for the betatron motion to become coupled if the beam ellipse is tilted in the $x-z$ plane. This manifests itself by the appearance of a non-zero tilt angle $\Theta$ of the half axes $E_{1}$, $E_{2}$ with respect to the $\mathrm{x}$ and $\mathrm{z}$ axes.

If the beam is injected with no twist, the ellipsoid can be written in the form [9] :

$$
\begin{aligned}
\overrightarrow{\hat{y}}\left(s_{0} ; \varphi, \chi, \delta_{I}, \delta_{I I}\right)= & \cos \varphi \cdot \cos \chi \cdot\left[\left(\begin{array}{c}
E_{x}\left(s_{0}\right) \\
E_{p_{x}}\left(s_{0}\right) \\
0 \\
0 \\
0
\end{array}\right) \cdot \cos \delta_{I}+\left(\begin{array}{c}
0 \\
\epsilon_{x} / E_{x}\left(s_{0}\right) \\
0 \\
0 \\
0
\end{array}\right) \cdot \sin \delta_{I}\right]+ \\
& \left.\cos \varphi \cdot \sin \chi \cdot\left(\begin{array}{c}
0 \\
0 \\
0 \\
0 \\
E_{z}\left(s_{0}\right) \\
E_{p_{z}}\left(s_{0}\right) \\
0
\end{array}\right) \cdot \cos \delta_{I I}+\left(\begin{array}{c}
E_{z}\left(s_{0}\right) \\
0 \\
0 \\
0 \\
0 \\
0 \\
(\Delta p / p)_{0}
\end{array}\right) \cdot \sin \delta_{I I}\right]+
\end{aligned}
$$

For open ended transport systems, there is little more to be said. But for storage rings we wish the ellipsoid to be periodic. This then means that the transfer matrix is periodic:

$$
\underline{\hat{M}}\left(s_{0}+L, s_{0}\right)=\underline{\hat{M}}\left(s_{0}+2 L, s_{0}+L\right)
$$

One may find the periodic ellipsoid by writing (3.4) in the form [9]

$$
\overrightarrow{\hat{y}}\left(s_{0} ; \varphi, \chi, \delta_{I}, \delta_{I I}\right)=\frac{1}{2} \cdot \sqrt{\varepsilon_{I}} \cdot \cos \varphi \cdot \cos \chi \cdot\left[\left(\begin{array}{c}
\vec{v}_{I}\left(s_{0}\right) \\
0
\end{array}\right) \cdot e^{i \delta_{I}}+\left(\begin{array}{c}
\vec{v}_{I}^{*}\left(s_{0}\right) \\
0
\end{array}\right) \cdot e^{-i \delta_{I}}\right]+
$$




$$
\begin{aligned}
& \frac{1}{2} \cdot \sqrt{\varepsilon_{I I}} \cdot \cos \varphi \cdot \sin \chi \cdot\left[\left(\begin{array}{c}
\vec{v}_{I I}\left(s_{0}\right) \\
0
\end{array}\right) \cdot e^{i \delta_{I I}}+\left(\begin{array}{c}
\vec{v}_{I I}^{*}\left(s_{0}\right) \\
0
\end{array}\right) \cdot e^{-i \delta_{I I}}\right]+ \\
& \sin \varphi \cdot\left(\begin{array}{c}
\vec{y}_{5}\left(s_{0}\right) \\
(\Delta p / p)_{0}
\end{array}\right)
\end{aligned}
$$

with

$$
\begin{array}{r}
\sqrt{\varepsilon_{I}} \cdot \vec{v}_{I}\left(s_{0}\right)=\vec{y}_{1}\left(s_{0}\right)-i \cdot \vec{y}_{2}\left(s_{0}\right) ; \\
\sqrt{\varepsilon_{I I}} \cdot \vec{v}_{I I}\left(s_{0}\right)=\vec{y}_{3}\left(s_{0}\right)=i \cdot \vec{y}_{4}\left(s_{0}\right) ; \\
\vec{v}_{I}^{+}\left(s_{0}\right) \cdot \underline{S} \cdot \vec{v}_{I}\left(s_{0}\right)=\vec{v}_{I I}^{+}\left(s_{0}\right) \cdot \underline{S} \cdot \vec{v}_{I I}\left(s_{0}\right)=i .
\end{array}
$$

From this, it is clear that an ellipsoid injected at $s=s_{0}$, recovers its original form after one turn if the vectors $\vec{v}_{I}\left(s_{0}\right)$ and $\vec{v}_{I I}\left(s_{0}\right)$ satisfy the eigen-condition

$$
\begin{aligned}
\underline{M}\left(s_{0}+L, s_{0}\right) \cdot \vec{v}_{I}\left(s_{0}\right) & =e^{-i ; 2 \pi Q_{I}} \cdot \vec{v}_{I}\left(s_{0}\right) ; \\
\underline{M}\left(s_{0}+L, s_{0}\right) \cdot \vec{v}_{I I}\left(s_{0}\right) & =e^{-i \cdot 2 \pi Q_{I I}} \cdot \vec{v}_{I I}\left(s_{0}\right)
\end{aligned}
$$

and when $\vec{y}_{5}\left(s_{0}\right)$ satisfies the condition

$$
\hat{\underline{M}}\left(s_{0}+L, s_{0}\right)\left(\begin{array}{c}
\vec{y}_{5}\left(s_{0}\right) \\
(\Delta p / p)_{0}
\end{array}\right)=\left(\begin{array}{c}
\vec{y}_{5}\left(s_{0}\right) \\
(\Delta p / p)_{0}
\end{array}\right) .
$$

Eqn.(4.15a,b) can be written as:

$$
\underline{\hat{M}}\left(s_{0}+L, s_{0}\right) \cdot \overrightarrow{\hat{u}}_{\nu}\left(s_{0}\right)=\lambda_{\nu} \cdot \overrightarrow{\hat{u}}_{\nu}\left(s_{0}\right)
$$

with

$$
\begin{aligned}
& \overrightarrow{\hat{u}}_{1}\left(s_{0}\right)=\left(\begin{array}{c}
\vec{v}_{I}\left(s_{0}\right) \\
0
\end{array}\right) ; \lambda_{1}=e^{-i \cdot 2 \pi Q_{I}} ; \\
& \overrightarrow{\hat{u}}_{2}\left(s_{0}\right)=\left(\begin{array}{c}
\vec{v}_{I I}\left(s_{0}\right) \\
0
\end{array}\right) ; \lambda_{2}=e^{-i \cdot 2 \pi Q_{I I}} ; \\
& \overrightarrow{\hat{u}}_{3}\left(s_{0}\right)=\left(\begin{array}{c}
\vec{y}_{5}\left(s_{0}\right) \\
(\Delta p / p)_{0}
\end{array}\right) ; \lambda_{3}=1 .
\end{aligned}
$$

Since the transfer matrix $\underline{\hat{M}}\left(s_{0}+L, s_{0}\right)$ depends on the eigenvectors $\overrightarrow{\hat{u}}_{\nu}\left(s_{0}\right)$ and its eigenvalues, the vectors $\overrightarrow{\hat{u}}_{\nu}\left(s_{0}\right)$ must be calculated self consistently by iteration: the calculation of $\underline{\hat{M}}$ in the $n$-th step relies on knowledge of the eigenvectors of the (n-1)-th step. In the first step the space charge forces are neglected. The solution so obtained will depend on the form for the starting ellipsoid and its charge density.

Since the tunes, $Q_{I}$ and $Q_{I I}$, depend on the eigenvalues $\lambda_{1}$ and $\lambda_{2}[7,8]$ (see eqn. $(4.17 \mathrm{a}, \mathrm{b})$ ), we are also able to obtain the tune shifts caused by the space charge forces.

As shown in Ref.[10], the Q shift can be approximately written in the form

$$
\delta Q_{k}=\frac{1}{2 \pi} \cdot \int_{s_{0}}^{s_{0}+L} d s \cdot\left[\vec{v}_{k}^{(0)}(s)\right]^{+} \cdot \underline{S} \cdot \delta \underline{A}(s) \cdot \vec{v}_{k}^{(0)}(s) ; \quad(k=I, I I),
$$


where $\delta \underline{A}(s)$ represents the perturbations due to space charge forces which appear in eqn. $(4.2 \mathrm{a})$ :

$$
\begin{aligned}
\delta A_{21} & =\frac{4 \lambda \cdot e}{\gamma^{3} \cdot m_{0} v^{2}} \cdot F_{x x} ; \\
\delta A_{23} & =\frac{4 \lambda \cdot e}{\gamma^{3} \cdot m_{0} v^{2}} \cdot F_{x z} \\
\delta A_{41} & =\frac{4 \lambda \cdot e}{\gamma^{3} \cdot m_{0} v^{2}} \cdot F_{z x} \\
\delta A_{43} & =\frac{4 \lambda \cdot e}{\gamma^{3} \cdot m_{0} v^{2}} \cdot F_{z z}
\end{aligned}
$$

and $\vec{v}_{k}^{(0)}$ are the eigenvectors of the unperturbed transfer matrix.

However, before eqn. (4.18) can be used for obtaining $Q$ shifts, the forces $F_{x x}, F_{x z}, F_{z x}$, $F_{z z}$ must be known. This will imply that the equilibrium ellipsoid is already known from iteration of eqn. (4.16) and that the Q-shifts have in any case already been obtained from the final eigentunes. Thus eqn. (4.18) offers no immediate practical advantage.

\section{Summary}

We have investigated the influence of space charge forces on the motion of charged particles in storage rings and transport systems.

In order to describe the bunch we have introduced a 5-dimensional ellipsoid in the $x-p_{x}-$ $z-p_{z}-\Delta p / p$ space represented by the "bunch-shape matrix", $\underline{\mathrm{B}}(\mathrm{s})$, which contains as columns, five independent orbit vectors. This matrix $\underline{B}(s)$ contains the complete information about the configuration of the bunch at the point $\mathrm{s}$ and can be obtained by matrix multiplication with the transfer matrix $\underline{\mathrm{M}}$.

In thin lens approximation the matrix takes a simple form which can be conveniently coded for computer.

In this report we have only considered the transverse betatron motion. The effect of space charge forces on longitudinal motion has, for example, been investigated by E.A.Karantzoulis and J.R.M.Maidment [5].

The use of the ellipsoid enables us to demonstrate that in general linear coupled systems, a bunch which has elliptical properties in the $x-z, x-p_{x}$ and $z-p_{z}$ plane will keep its ellipse-like projections in its subsequent motion.

The equations so derived could be used for studying beam transport in DESY III .

A generalization to the 6-dimensional case is in preparation.

\section{References}

[1] See e.g. Sard: Relativistic Mechanics; W. A. Benjamin, Inc.

New York(1970).

The effects of orbit curvature on interparticle forces have recently been discussed in:

R.Talman: "Novel relativistic effect important in accelerators" ; 
Phys.Rev.Lett. 56, p.1429 (1986).

A.Piwinski: "On the transverse forces caused by the curvature" ; CERN/LEP-TH/85-43.

M.Bassetti: "Analytical formula for the centrifugal space charge effects" ; CERN/LEP$\mathrm{TH} / 86-13$.

M.Bassetti, D.Brandt: "Transverse electromagnetic forces in circular trajectory" ; CERN/LEP-TH/86-04.

These effects are not considered in this paper.

[2] J.M.Kapchinskij and V.V.Vladimirskij, Conference on High-Energy Accelerators and Instrumentation, p.274, CERN Geneva (1959).

[3] Shoroku Ohnuma and Joseph N. Vitale: "Space Charge Effects on the Quadrupole Focusing System in Low-Energy Proton Linear Accelerators"; IEEE Transactions on Nuclear Science, p.594, 1967.

[4] F.J.Sacherer: "RMS Envelope Equations with Space Charge"; IEEE Transactions on Nuclear Science, NS-18,p.1105, 1971.

[5] E.A.Karantzoulis and J.R.M.Maidment: "Beam dynamics at low energy in DESY III"; DESY-HERA 86-01.

[6] D.P.Barber, G.Ripken, F.Schmidt: "A non-linear canonical formalism for the coupled synchro-betatron motion of protons with arbitrary energy"; DESY M-87-36.

[7] H.Mais, G.Ripken: "Theory of coupled Synchro-Betatron Oscillations I"; DESY M-82-17.

[8] H.Mais, G.Ripken: "Theory of Spin-Orbit Motion in Electron-Positron Storage Rings"; DESY 83-062.

[9] G.Ripken: "Untersuchungen zur Strahlfuehrung und Stabilitaet der Teilchenbewegung in Beschleunigern und Storage-Ringen unter strenger Beruecksichtigung einer Kopplung der Betatronschwingungen"; DESY R1-70/04.

[10] H. Mais, G. Ripken: "Influence of the synchrotron radiation on the spin-orbit motion of a particle in a storage ring"; DESY M-82-20.

\section{Acknowledgements}

We wish to thank Dr. D. P. Barber for many stimulating discussions and guidance, for helping to translate the text and for careful reading of the manuscript. We also thank Prof. Dr. B. Wiik for continued encouragement and Dr. Y. H. Chin and Dr. F. Willeke for helpful and interesting discussions. 